\title{
Review Article \\ Oxidative Stress-Related Biomarkers in Postmenopausal Osteoporosis: A Systematic Review and Meta-Analyses
}

\author{
Qiaozhen Zhou, ${ }^{1}$ Li Zhu, ${ }^{1}$ Dafeng Zhang, ${ }^{1}$ Ning Li, ${ }^{1}$ Qiao Li, ${ }^{2}$ Panpan Dai, ${ }^{1,3}$ Yixin Mao, ${ }^{1,3}$ \\ Xumin $\mathrm{Li}^{1,3}$ Jianfeng Ma, ${ }^{1,3}$ and Shengbin Huang ${ }^{1,3}$ \\ ${ }^{1}$ Department of Prosthodontics, School and Hospital of Stomatology, Wenzhou Medical University, Wenzhou 325027, China \\ ${ }^{2}$ Department of Pediatric Dentistry, School and Hospital of Stomatology, Wenzhou Medical University, Wenzhou 325027, China \\ ${ }^{3}$ Institute of Stomatology, School and Hospital of Stomatology, Wenzhou Medical University, Wenzhou 325027, China
}

Correspondence should be addressed to Jianfeng Ma; dentistmacn@aliyun.com and Shengbin Huang; huangsb003@wmu.edu.cn

Received 5 April 2016; Accepted 3 July 2016

Academic Editor: Fabrizia Bamonti

Copyright (C) 2016 Qiaozhen Zhou et al. This is an open access article distributed under the Creative Commons Attribution License, which permits unrestricted use, distribution, and reproduction in any medium, provided the original work is properly cited.

\begin{abstract}
Numerous studies suggested that oxidative stress (OS) played a central role in the onset and development of postmenopausal osteoporosis (PO); however, conflicting results were obtained as to the association of OS-related biomarkers and PO. This metaanalysis aimed to identify the association between these markers and PO, and explore factors that may explain the inconsistencies in these results. A systematic literature search was conducted in relevant database. Search terms and selection criteria were priorly determined to identify and include all studies that detected markers of OS in PO patients. We pooled data with a random effects meta-analysis with standardized mean differences and 95\% confidence interval. Total 17 studies including 12 OS markers were adopted. The results showed that superoxide dismutase (SOD) in erythrocytes, catalase (CAT), total antioxidant status (TAS), hydroperoxides (HY), advanced oxidation protein products (AOPP), malondialdehyde (MDA), and vitamin B12 $\left(\mathrm{VB}_{12}\right)$ in plasma/serum were not statistically different between the $\mathrm{PO}$ and control group, whereas significantly increased level of homocysteine (Hcy) and nitric oxide (NO), along with decreased SOD, glutathione peroxidase (GPx), folate, and total antioxidant power (TAP) in plasma/serum were obtained in the PO group. In summary, OS might serve as potential biomarkers in the etiopathophysiology and clinical course of PO.
\end{abstract}

\section{Introduction}

Postmenopausal osteoporosis (PO) is one of the most common bone diseases, characterized by low bone mineral density (BMD) and pathological fracture, which leads to significant morbidity [1]. Surgeon General's report (2004) on bone health and osteoporosis revealed that osteoporosis affected more than 8 million women and 2 million men in the USA, in addition to 34 million people with low bone mass [2]. These numbers are expected to increase steadily over time, with osteoporosis affecting an estimated 14 million people and low bone mass affecting about 48 million people by the year 2020 [3]; thus, early diagnosis, prevention, and treatment of osteoporosis [4] are extremely important. However, population screening by dual-energy X-ray absorptiometry (DXA) bone scans (which is the current gold standard for osteoporosis diagnosis) is not cost-effective; new and reliable methods are required to identify individuals with low BMD [5].

In spite of the remarkable progress achieved in the understanding of how estrogen deficiency induces PO, the underlying pathogenic mechanisms have been found to be complex and multifaceted [1]. One of the most intriguing hypotheses is the ability of these sexual hormones to protect bone against OS by acting as an antioxidant [6]. Moreover, a senior researcher proposed a paradigm shift from the "estrogencentric" account of the pathogenesis to one where OS was also involved in the development of osteoporosis [7]. This further emphasizes the centric role of OS in the onset and development of PO.

OS is generated as a result of insufficient activity of the endogenous antioxidant defense system against reactive oxygen species (ROS). On the one hand, excessive ROS are able to 
exert oxidative damage to lipids, protein, and DNA [8], which yield relatively stable oxidized biomolecule products: MDA, protein carbonyls, 3-nitrotyrosine, 8-hydroxyguanosine (8$\mathrm{OHG}$ ), and so on. On the other hand, the antioxidant levels (vitamins E, C, A, and B6 and folate) and antioxidant enzyme activity (SOD, CAT, and GPx) significantly decreased although the expression levels of some of them were also increased in the OS-related disease. Thus, either enhanced ROS production or impaired antioxidant system will tip the cellular redox balance to oxidative imbalance and cause ROS overproduction [9].

Experimental studies demonstrated that OS is an important factor in bone remodeling [10-12]. The results have been further shown by cross-sectional and case-control studies, in which OS was characterized by a high level of $\mathrm{F}_{2}$-isoprostanes in urine and a low level of antioxidant enzymes in blood, along with a reduced bone mineral density and an increased risk of osteoporosis [13, 14]. In line with these findings, decreased SOD activity $[15,16]$ has been found in postmenopausal women compared with healthy controls, while other researchers revealed that there was no significant change in SOD activity in these patients $[17,18]$. These discrepancies might be due to differences in laboratory methods, biological specimens used for OS, or the extent to which studies took potential confounders such as health and lifestyle factors into account.

In addition, there is a wide range of OS biomarkers and laboratory techniques available, each of which has its own strength and limitation [19]. It is difficult to make measurement of ROS due to its short half-life. Levels of antioxidants, vitamins, or antioxidant enzymes are informative; however, they only reflect one side of the redox homeostasis, leaving the question of whether decreased levels are actually also indicative of increased oxidative damage unanswered [20]. Currently, there is no consensus on the most appropriate biomarkers of OS for PO and the validity of many of the biomarkers in use needs to be confirmed. Thus, we aimed to perform a meta-analysis to quantitatively assess all the published clinical trials to determine whether there is an association between OS and the development of PO and, meanwhile, examine whether the OS-related biomarkers could be regarded as potential diagnostic/prognostic markers of PO in clinical application.

\section{Methods}

2.1. Search Strategy. Identification of the studies was carried out through an extensive literature search using the PubMed database, ISI Web of Science, EMBASE, and Google Scholar mainly based on the search terms, with English restriction, and updated to February 2016. The search strategy included the terms "oxidative stress", "bone mineral density", and "postmenopausal osteoporosis" and they were used in text word searches, and the "related articles" function was used to broaden the search. Publications cited in references found using these search terms were also reviewed for any relevant studies, which were not already identified; in addition, all searches were conducted prior to February 2016 with no time span specified.
2.2. Study Selection and Data Extraction. We searched all abstracts for potentially relevant publications. Studies meeting the following criteria were included: (1) having measured levels of one or more of the following OS markers in both PO patients and healthy controls: SOD, CAT, Hcy, GPx, protein carbonyl, 3-nitrotyrosine, NO, vitamins, folate, lipid peroxidation, TAP, and TAS; (2) being reported in an original research paper in a peer-reviewed journal; and (3) adequately describing their samples (e.g., diagnostic criteria, source of samples, and storage) and methods such that the experiments could be replicated (or included appropriate references). For all included studies, the study design, sample type, age, and BMI of each group and biomarkers of interest were recorded.

Papers were excluded (1) if the study only enrolled subjects with postmenopausal osteoporosis; (2) if the outcomes were not reported as the mean \pm standard deviation (SD); (3) if the BMD was not evaluated by dual-energy X-ray absorptiometry (DXA); and (4) if postmenopausal women took estrogen replacement therapy (ERT) before the clinical trial. Finally, studies were checked carefully to ensure that the diagnosis criteria of PO were similar among the studies. When several reports from the same study were published, only the most recent or informative one was included in our meta-analysis. Only biomarkers that were the object of at least 2 independent studies were included.

To reduce the heterogeneity, the studies included in the meta-analysis were only carried out on the same biological sample, except for plasma and serum. All the studies had a cross-sectional design, with cases mostly diagnosed according to the BMD T-score (the number of standard deviations below the average for a young adult at peak bone density) lower than 2.5 standard deviations from BMD peak at either femoral neck or lumbar spine, according to WHO guidelines.

2.3. Statistical Analysis. The meta-analysis was conducted using Stata statistical software version 12.1 (Stata, College Station, TX, USA). Standardized mean differences were used to construct forest plots of continuous data. $P<0.05$ was considered statistically significant, and $95 \%$ confidence intervals (CIs) were reported. A random-effects model was used and studies were weighted by the generic inverse variance method ( $Q$ statistic: $P<0.10, I^{2}>50 \%$ ). If a sufficient number of trials (more than 10) were included in any meta-analysis, publication bias was to be assessed according to the recommendations on testing for funnel plot asymmetry as described in the Cochrane Handbook. One-study removed sensitivity analysis was performed for each oxidation marker to determine robustness by manually excluding each study included in the analysis.

\section{Results}

3.1. Literature Search. The selection of literature for included studies was shown in Figure 1. A total of 704 potential records were identified from the databases, with 688 studies excluded. 17 articles $[6,15,18,21-34]$ fulfilled selection criteria and were illustrated in detail in Table 1 . All the studies had a cross-sectional design, with cases mostly diagnosed according to the following criteria: osteoporosis T-score is 


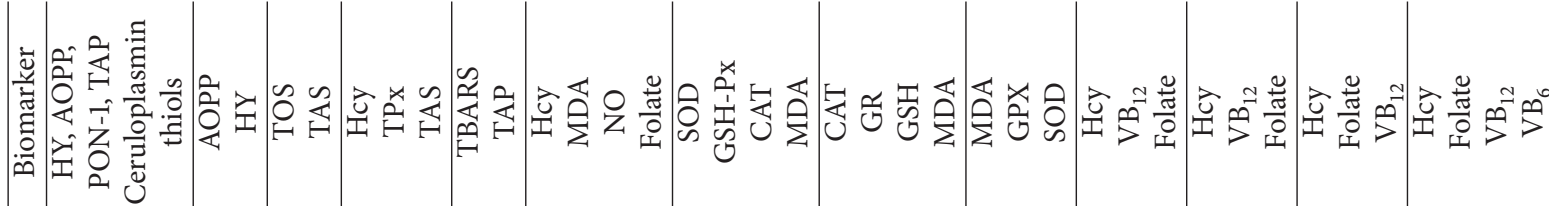

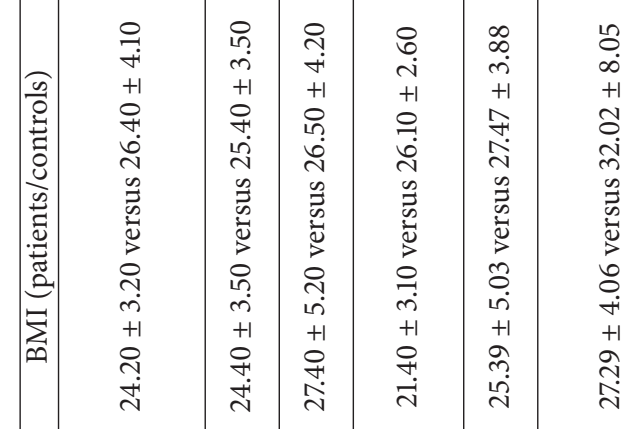

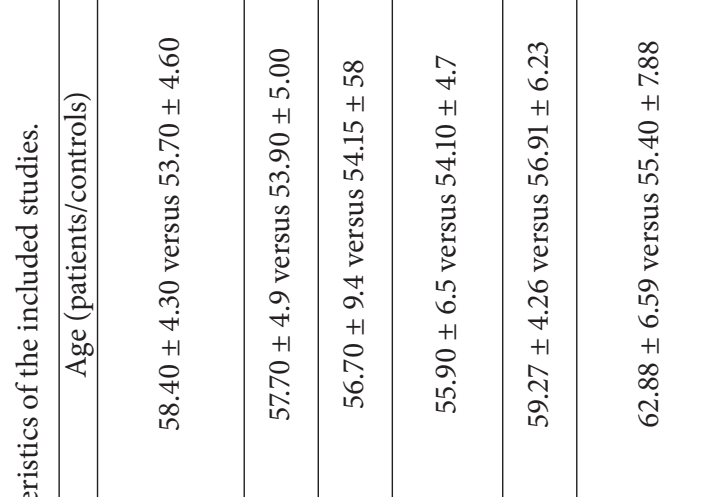

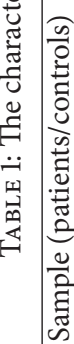

|

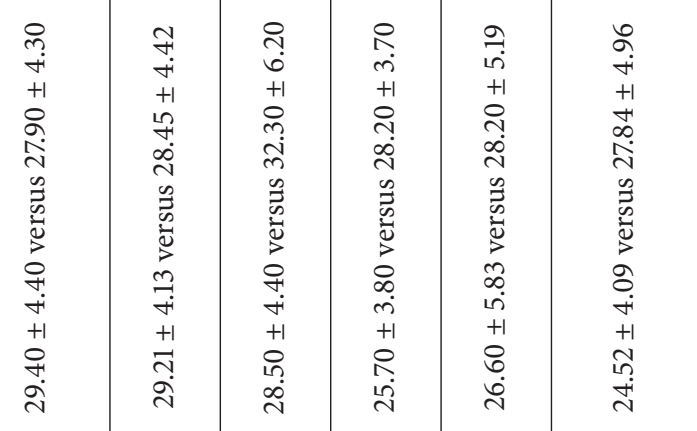

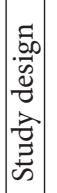

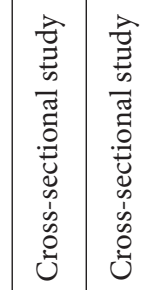

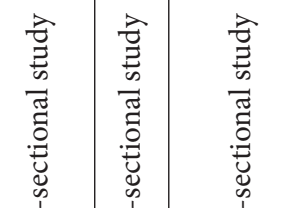

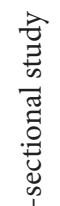

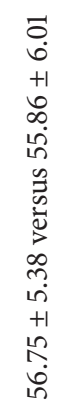

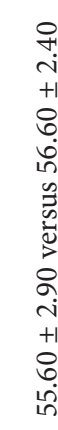

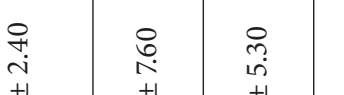

ஃ

A

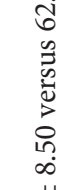

0
0
5
5
0
0
10
$\infty$
$\infty$
+
0

in

离

รั)

+2
+1
+1

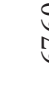

के

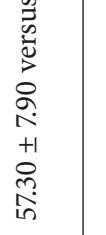

in

हि

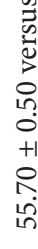

웅

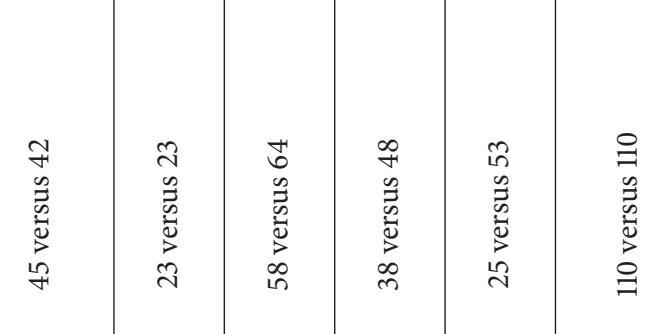

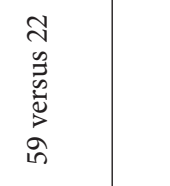

Uु

它

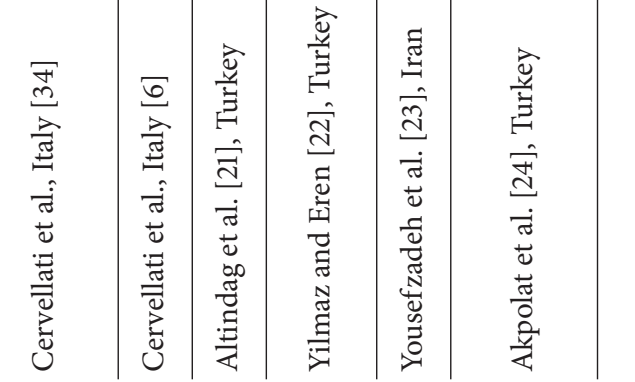

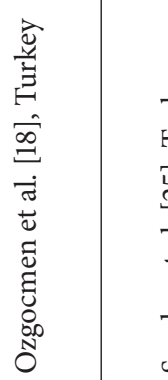

离

莪

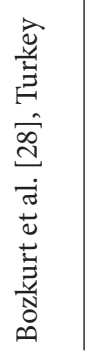

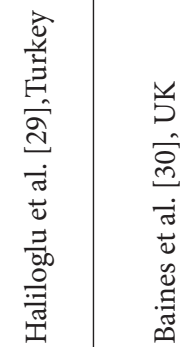




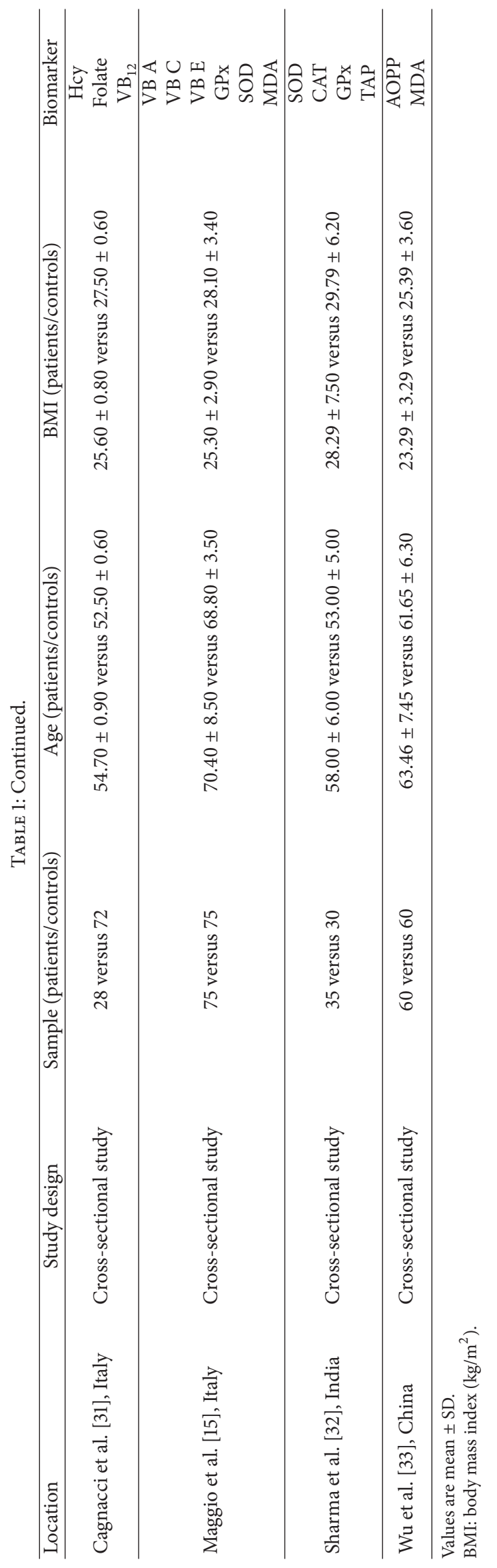


TABLE 2: The relationship between enzymatic antioxidant and risk of PO.

\begin{tabular}{|c|c|c|c|c|c|c|}
\hline \multirow{2}{*}{ First author } & \multirow{2}{*}{ Biomarker } & \multirow{2}{*}{ Biologic sample } & \multirow{2}{*}{ Sample (patients/controls) } & \multirow{2}{*}{$\begin{array}{c}\text { SMD } \\
(95 \% \mathrm{CI})\end{array}$} & \multicolumn{2}{|c|}{ Heterogeneity } \\
\hline & & & & & $I^{2}$ & $P$ value \\
\hline Ozgocmen [18] & CAT & Erythrocytes (fasting) & 59 versus 22 & $-1.82(-2.39,-1.26)$ & & \\
\hline Sendu [25] & CAT & Erythrocytes & 45 versus 42 & $-0.30(-0.72,0.12)$ & & \\
\hline $\operatorname{SMD}(95 \% C I)$ & $C A T$ & Erythrocytes & 104 versus 64 & $-1.05(-2.54,0.44)$ & $94.4 \%$ & $<0.0001$ \\
\hline Maggio [15] & SOD & Erythrocyte & 75 versus 75 & $-2.70(-3.14,-2.26)$ & & \\
\hline Ozgocmen [18] & SOD & Erythrocytes (fasting) & 59 versus 22 & $0.16(-0.33,0.65)$ & & \\
\hline $\operatorname{SMD}(95 \% C I)$ & SOD & Erythrocytes & 134 versus 97 & $-1.27(-4.07,1.53)$ & $98.6 \%$ & $<0.0001$ \\
\hline Sharma [32] & SOD & Serum & 35 versus 30 & $-4.03(-4.89,-3.17)$ & & \\
\hline Maggio [15] & SOD & Plasma & 75 versus 75 & $-2.05(-2.45,-1.66)$ & & \\
\hline $\operatorname{SMD}(95 \% \mathrm{CI})$ & $S O D$ & Plasma/serum & 110 versus 105 & $-3.00(-4.94,-1.07)$ & $94.1 \%$ & $<0.0001$ \\
\hline Maggio [15] & GPx & Plasma & 75 versus 75 & $-2.00(-2.39,-1.61)$ & & \\
\hline Sharma [32] & GPx & Serum & 35 versus 30 & $-5.51(-6.59,-4.43)$ & & \\
\hline $\operatorname{SMD}(95 \% C I)$ & $G P x$ & Plasma/serum & 110 versus 105 & $-2.41(-2.78,-2.04)$ & $97.2 \%$ & $<0.0001$ \\
\hline
\end{tabular}

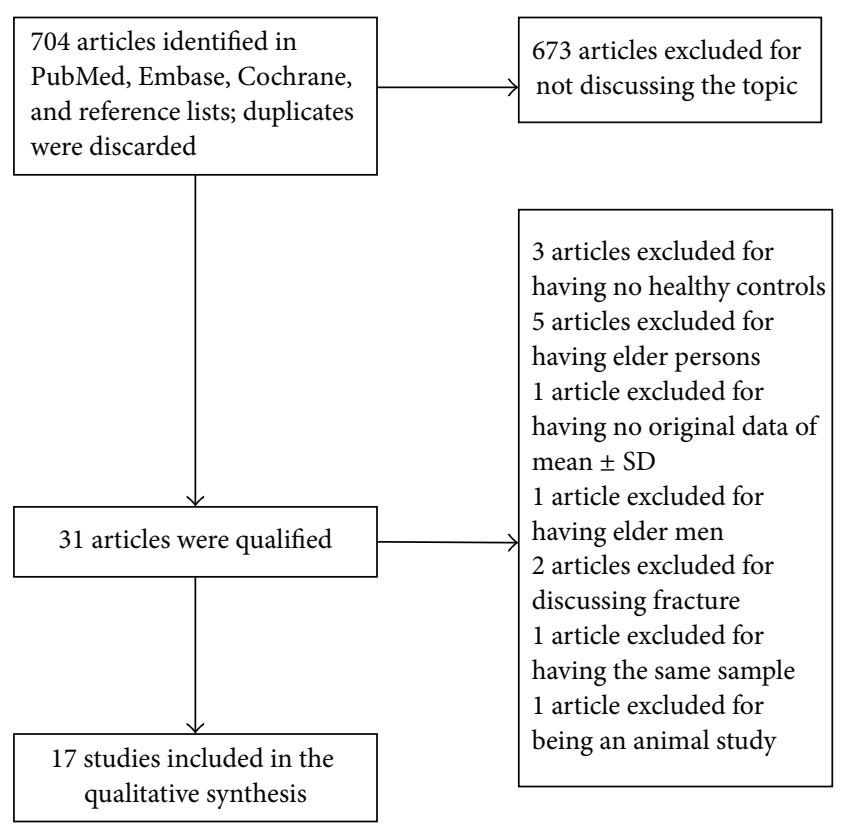

FIGURE 1: Search strategy flow diagram.

more than 2.5 SD below peak bone mass. Totally, 12 OS markers were included in this meta-analysis. Six out of these twelve OS markers showed a statistically significant change in PO patients compared to healthy controls: GPx, SOD, Hcy, NO, folate, and TAP, while no statistical difference was found regarding the other biomarkers. Forest plots of all standardized mean differences and $95 \%$ confidence intervals were shown in Figures 2-5. Figure 2 was the result of forest plot of meta-analysis of the relationship between enzymatic antioxidant and risk of PO, while Figures 3-5 showed the relationship of free radicals products/antioxidants and risk of PO for MDA, AOPP/HY, NO, $\mathrm{VB}_{12}$, folate, and Hcy. The relationships between oxidative stress-related biomarkers and the risk of $\mathrm{PO}$ were shown in Tables 2 and 3.
All the identified papers were published between 2003 and 2016. Eight studies [18, 21, 22, 24-26, 28, 29] were conducted in Turkey, $4[6,15,31,34]$ were from Italy, and the others were from India [32], UK [30], Morocco [27], Iran [23], and China [33], respectively. The study size was relatively small with the number of cases ranging from 22 to 110 and the number of controls from 15 to 110 .

3.2. The Relationship between Enzymatic Antioxidant and Risk of PO. The levels of antioxidant enzymes in PO cases and controls were reported in 12 articles.

3.2.1. CAT. CAT activity in erythrocytes was measured in 2 papers $[18,25]$. After meta-analysis, no statistically decreased level of CAT in PO was found when compared with that in controls group $(-1.05,95 \% \mathrm{CI}-2.54-0.44, P>0.05)$.

3.2.2. SOD. SOD activity was measured in 3 papers $[15,18$, 32]. The SOD activity assessed in erythrocytes was -1.27 (95\% CI $-4.07-1.53)$ and -3.00 (95\% CI -4.94 to -1.07$)$ in serum and plasma, respectively. After meta-analysis, a statistically decreased level of SOD activity in serum/plasma was obtained in the PO group $(P<0.05)$, while no significant difference was found related to the SOD activity in erythrocytes.

3.2.3. GPx. GPx activity was reported in 3 papers, while 2 studies reported on GPx activity in the plasma/serum samples. After meta-analysis, a significantly lower GPx activity was found in PO subjects than that in controls $(-3.72,95 \%$ CI -7.16 to -0.28$),(P<0.05)$. This was in accordance with the result obtained in erythrocytes.

3.2.4. TAP. The meta-analysis including 4 trials with 408 subjects revealed that TAP level was significantly decreased in the PO group compared to the control group under a random-effects model $(-2.74,95 \% \mathrm{CI}-4.60-1.08)$. 


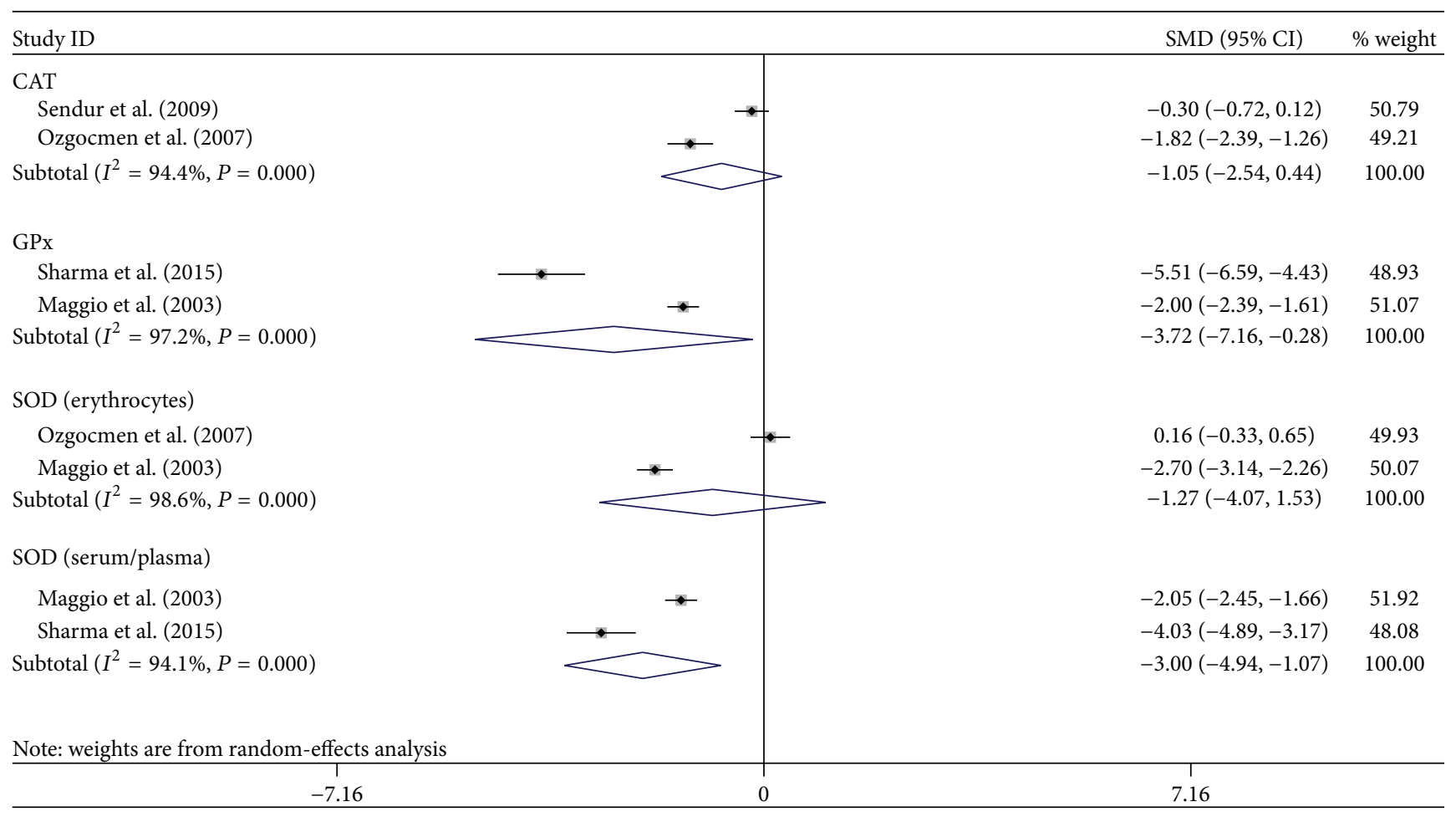

FIGURE 2: Forest plot of meta-analysis of the relationship between enzymatic antioxidant and risk of PO.

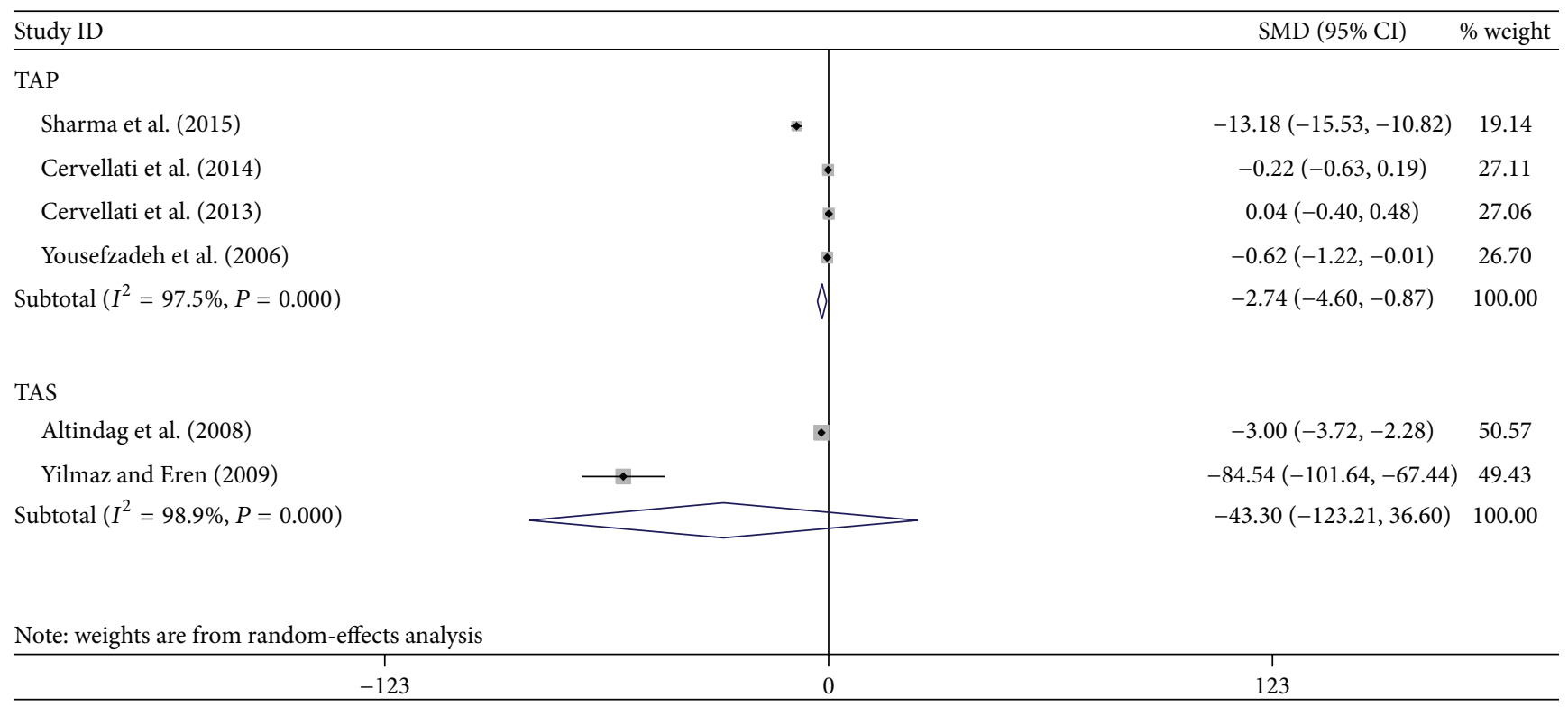

FIGURE 3: Forest plot of meta-analysis of the relationship between TAP/TAS and risk of PO.

3.2.5. TAS. In the present study, the meta-analysis including 2 trials with 124 subjects revealed that, with regard to TAS level, there was no statistical difference between PO group and control group under a random-effects model $(-43.30,95 \% \mathrm{CI}$ $-123.21-36.60)$.

\subsection{The Relationship of Free Radicals Products and Risk of PO}

3.3.1. MDA. A forest plot that provided suitable data for statistical pooling revealed that there was no significant difference obtained for MDA levels between PO group and control group $(0.50,95 \%$ CI $-0.08-1.08)$. 


\begin{tabular}{|c|c|c|c|}
\hline Study ID & & SMD $(95 \% \mathrm{CI})$ & $\%$ weight \\
\hline MDA & & & \\
\hline Akpolat et al. (2013) & $\rightarrow$ & $1.15(0.78,1.53)$ & 24.96 \\
\hline Maggio et al. (2003) & E & $-0.16(-0.48,0.16)$ & 25.71 \\
\hline Sendur et al. (2009) & - & $0.75(0.31,1.19)$ & 24.13 \\
\hline Wu et al. (2015) & 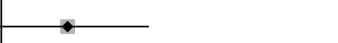 & $0.29(-0.07,0.65)$ & 25.20 \\
\hline Subtotal $\left(I^{2}=89.9 \%, P=0.000\right)$ & & $0.50(-0.08,1.08)$ & 100.00 \\
\hline AOPP & & & \\
\hline Wu et al. (2015) & $\longrightarrow$ & $1.07(0.68,1.45)$ & 33.94 \\
\hline Cervellati et al. (2013) & - & $0.14(-0.29,0.58)$ & 32.77 \\
\hline Cervellati et al. (2014) & 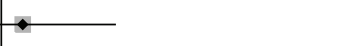 & $0.09(-0.32,0.51)$ & 33.29 \\
\hline Subtotal $\left(I^{2}=86.3 \%, P=0.001\right)$ & $=$ & $0.44(-0.20,1.08)$ & 100.00 \\
\hline HY & & & \\
\hline Cervellati et al. (2013) & 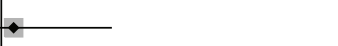 & $0.05(-0.38,0.49)$ & 47.52 \\
\hline Cervellati et al. (2014) & $\bullet$ & $0.27(-0.14,0.68)$ & 52.48 \\
\hline Subtotal $\left(I^{2}=0.0 \%, P=0.481\right)$ & $>$ & $0.17(-0.13,0.47)$ & 100.00 \\
\hline NO & & & \\
\hline Sendur et al. (2009) & $\rightarrow$ & $0.60(0.17,1.03)$ & 41.33 \\
\hline Akpolat et al. (2013) & $\longrightarrow$ & $0.72(0.36,1.09)$ & 58.67 \\
\hline Subtotal $\left(I^{2}=0.0 \%, P=0.674\right)$ & & $0.67(0.40,0.95)$ & 100.00 \\
\hline Note: weights are from random-effect & & & \\
\hline $\begin{array}{c} \\
-1.53\end{array}$ & 0 & $\begin{array}{c}1 \\
1.53\end{array}$ & \\
\hline
\end{tabular}

FIGURE 4: Forest plot of meta-analysis of the relationship of free radicals products and risk of PO.

3.3.2. AOPP and HY. From the results, we could find that only 3 papers measured the AOPP and 2 papers measured the HY activity in PO. The final results of meta-analyses showed that no significantly higher AOPP $(0.44,95 \% \mathrm{CI}$ $-0.20-1.08)$ and HY $(0.17,95 \% \mathrm{CI}-0.13-0.47)$ appeared in the PO subjects.

3.3.3. NO. Meta-analysis of 2 trials with 168 subjects revealed that the NO level was statistically higher in the PO group than in the control group under a random-effects model $(0.67,95 \%$ CI 0.40-0.95).

\subsection{The Relationship of Nutrient Status and Risk of PO}

3.4.1. $V B_{12}$. A total of 5 studies reported results on $\mathrm{VB}_{12}$. All the separated papers found no statistical difference between cases and controls after combining all the raw data. The metaanalysis also showed no statistically decreased $\mathrm{VB}_{12}$ level in the PO group than in the control group under a randomeffects model $(0.00,95 \%$ CI $-0.20-0.21)$.

3.4.2. Folate. Folate activity was evaluated in 6 studies. The heterogeneity was significant $\left(P<0.0001, I^{2}=96.4 \%\right)$. A meta-analysis of 6 trials with 732 participants revealed that the folate level statistically decreased in the PO under a random-effects model $(-1.18,95 \% \mathrm{CI}-2.04$ to -0.31$)$.
3.4.3. Hcy. Seven papers on Hcy were adopted in the current meta-analysis. The heterogeneity was significant $(P=0.08$, $\left.I^{2}=75.3 \%\right)$. The results of the meta-analysis of the trials with 781 participants revealed that the Hcy level was significantly higher in the PO than that in the control group under a random-effects model $(0.53,95 \%$ CI $0.23-0.84)$.

3.5. Sensitivity Analysis and Publication Bias. Given the small number of studies, we performed a one-study removed sensitivity analysis by excluding each study individually. The effect size of MDA, AOPP, TAP, $\mathrm{VB}_{12}$, folate, and Hcy remained essentially unchanged in direction and magnitude after the removal of each study individually. We intended to assess publication bias, but the studies for each outcome of interest were too few to derive meaning from funnel plots.

\section{Discussion}

To our knowledge, this is the first meta-analysis to clarify and quantify the relationship between OS-related biomarkers and $\mathrm{PO}$ patients. Our research further supported the presence of oxidative damage in PO patients. The results showed increased Hcy and NO in the PO subjects, while it showed decreased levels of folate and TAP, along with lower activity of SOD and GPx in these subjects. 


\begin{tabular}{|c|c|c|c|}
\hline Study ID & & SMD $(95 \%$ CI $)$ & $\%$ weight \\
\hline \multicolumn{4}{|l|}{ VB12 } \\
\hline Ouzzif et al. (2012) & $\rightarrow$ & $-0.13(-0.48,0.23)$ & 21.78 \\
\hline Bozkurt et al. (2009) & - & $0.05(-0.37,0.48)$ & 16.84 \\
\hline Haliloglu et al. (2010) & $\rightarrow$ & $-0.19(-0.66,0.29)$ & 14.15 \\
\hline Baines et al. (2007) & + & $-0.08(-0.34,0.19)$ & 31.29 \\
\hline Cagnacci et al. (2003) & $\leftarrow$ & $0.46(0.02,0.90)$ & 15.94 \\
\hline Subtotal $\left(I^{2}=29.6 \%, P=0.224\right)$ & $\phi$ & $0.00(-0.20,0.21)$ & 100.00 \\
\hline \multicolumn{4}{|l|}{ Folate } \\
\hline Akpolat et al. (2013) & $\vdash$ & $-1.03(-1.40,-0.66)$ & 17.07 \\
\hline Ouzzif et al. (2012) & $\rightarrow$ & $-0.24(-0.60,0.11)$ & 17.11 \\
\hline Bozkurt et al. (2009) & + & $0.04(-0.39,0.46)$ & 16.90 \\
\hline Haliloglu et al. (2010) & $\rightarrow-$ & $-0.46(-0.94,0.02)$ & 16.71 \\
\hline Baines et al. (2007) & $\leftarrow$ & $-0.39(-0.66,-0.12)$ & 17.33 \\
\hline Cagnacci et al. (2003) & $\longrightarrow$ & $-5.52(-6.41,-4.64)$ & 14.87 \\
\hline Subtotal $\left(I^{2}=96.4 \%, P=0.000\right)$ & & $-1.18(-2.04,-0.31)$ & 100.00 \\
\hline \multicolumn{4}{|l|}{ Hcy } \\
\hline Yilmaz and Eren (2009) & $\rightarrow$ & $0.25(-0.36,0.86)$ & 11.12 \\
\hline Akpolat et al. (2013) & $\rightarrow$ & $0.09(-0.26,0.44)$ & 15.69 \\
\hline Ouzzif et al. (2012) & $\rightarrow$ & $0.51(0.14,0.87)$ & 15.49 \\
\hline Bozkurt et al. (2009) & $\rightarrow$ & $0.43(-0.00,0.86)$ & 14.21 \\
\hline Haliloglu et al. (2010) & $\rightarrow-$ & $1.15(0.64,1.65)$ & 12.80 \\
\hline Baines et al. (2007) & 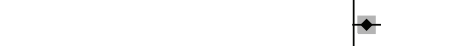 & $0.24(-0.03,0.50)$ & 17.17 \\
\hline Cagnacci et al. (2003) & $\rightarrow$ & $1.22(0.75,1.68)$ & 13.52 \\
\hline Subtotal $\left(I^{2}=75.3 \%, P=0.000\right)$ & $\diamond$ & $0.53(0.23,0.84)$ & 100.00 \\
\hline \multicolumn{4}{|c|}{ Note: weights are from random-effects analysis } \\
\hline $\begin{array}{cc} & \\
& -6.41 \\
\end{array}$ & 0 & $\begin{array}{c}1 \\
6.41\end{array}$ & \\
\hline
\end{tabular}

FIGURE 5: Forest plot of meta-analysis of the relationship of nutrient status and risk of PO.

ROS are usually highly reactive and unstable and have a very short half-life, thus making them difficult to measure directly. Oxidized biomolecule products generated by ROS are much more stable and commonly used as ROS markers. In addition, ROS could also be assessed indirectly by measuring antioxidant levels or antioxidant enzymes activity [35]. Thus, in this meta-analysis, both markers of OS products and systemic antioxidant capacity had been extensively evaluated.

Antioxidant system would stop the radical chain reaction and direct the resultant ROS to target where it would cause less injury [36]. Thus, three main enzymes responsible for ROS control, SOD, GPx, and CAT $[37,38]$, were adopted in the current meta-analysis. From the results, we clearly found that the activity of CAT and SOD in erythrocytes did not show any significant changes in PO patients as compared to healthy controls, while SOD activity in plasma/serum sample exhibited a decreased trend. This inconsistent result was due to the different sample source. Consistent with the result of SOD in plasma/serum, GPx activity also decreased significantly, which was also reflected by the decrease in TAP. From this perspective, our results seemed to be internally consistent. There was no significant difference in the existence of OS product: AOPP, HY, and MDA in PO, which was in line with the level of TAS. Thus, an important finding of this analysis was that the major changes in the redox balance relied on the deficits in plasma/serum antioxidants. However, on the one hand, OS is a dynamic and complex process [10]; thus, integral clinical interpretation should be considered concerning the parameter's abnormal values in further study. The percent ratio of the TOS to the TAS gave the oxidative stress index (OSI), an indicator of the degree of oxidative stress that may be used as an optional one. On the other hand, due to the limitation of detection methods on OS product and the highly reactive characters, more accurate methods will be needed in the future studies on examination of these biomarkers in PO.

Numerous researches suggested that $\mathrm{NO}$ acted as an important regulator on bone metabolism [36, 39, 40]. NO is a very unstable molecule, which makes direct quantitative measurement of NO in biological samples difficult. The authors detected the reaction using pretreatment of samples to reduce nitrate to nitrite, which can be accomplished by catalytic reactions using cadmium. Therefore, stable oxidation end products of $\mathrm{NO}$ can be readily measured in biological fluids. In the present study, 3 papers were included and the metaanalysis with 2 studies revealed that the NO level was statistically higher in the PO group compared to the control group. This further demonstrated the net effects of $\mathrm{NO}$ in the bone turnover of PO subjects.

Information about nutrient status of PO was also taken into consideration in this meta-analysis. Studies suggested 
TABLE 3: The relationship of free radicals products/antioxidants and risk of PO.

\begin{tabular}{|c|c|c|c|c|c|c|}
\hline \multirow{2}{*}{ First author } & \multirow{2}{*}{ Biomarker } & \multirow{2}{*}{ Biologic sample } & \multirow{2}{*}{ Sample (patients/controls) } & \multirow{2}{*}{$\begin{array}{c}\text { SMD } \\
(95 \% \mathrm{CI})\end{array}$} & \multicolumn{2}{|c|}{ Heterogeneity } \\
\hline & & & & & $I^{2}$ & $P$ value \\
\hline Sendur [25] & MDA & Plasma & 45 versus 42 & $0.75(0.31,1.19)$ & & \\
\hline Akpolat [24] & MDA & Serum & 66 versus 60 & $1.15(0.78,1.53)$ & & \\
\hline Maggio [15] & MDA & Plasma & 75 versus 75 & $-0.16(-0.48,0.16)$ & & \\
\hline $\mathrm{Wu}[33]$ & MDA & Plasma & 60 versus 60 & $0.29(-0.07,0.65)$ & & \\
\hline SMD $(95 \% C I)$ & $M D A$ & Plasma/serum & 246 versus 227 & $0.43(0.25,0.61)$ & $89.9 \%$ & $<0.0001$ \\
\hline $\mathrm{Wu}[33]$ & AOPP & Plasma & 60 versus 60 & $1.07(0.68,1.45)$ & & \\
\hline Cervellati [6] & AOPP & Serum & 30 versus 63 & $0.09(-0.32,0.51)$ & & \\
\hline Cervellati [34] & AOPP & Serum & 56 versus 38 & $0.14(-0.29,0.58)$ & & \\
\hline SMD (95\% CI) & $A O P P$ & Serum & 146 versus 158 & $0.44(-0.20,1.08)$ & $86.3 \%$ & 0.001 \\
\hline Cervellati [34] & HY & Serum & 56 versus 38 & $0.27(-0.14,0.68)$ & & \\
\hline Cervellati [6] & HY & Serum & 30 versus 63 & $0.05(-0.38,0.49)$ & & \\
\hline$S M D(95 \% C I)$ & $H Y$ & Serum & 96 versus 101 & $0.17(-0.13,0.47)$ & $0 \%$ & 0.48 \\
\hline Akpolat [24] & NO & Serum & 66 versus 60 & $0.72(0.36,1.09)$ & & \\
\hline Sendu [25] & NO & Plasma & 45 versus 42 & $0.60(0.17,1.03)$ & & \\
\hline$S M D(95 \% C I)$ & NO & Plasma/serum & 111 versus 102 & $0.67(0.40,0.95)$ & $0 \%$ & $>0.5$ \\
\hline Altindag [21] & TAS & Plasma & 39 versus 26 & $-3.00(-3.72,-2.28)$ & & \\
\hline Yilmaz [22] & TAS & Plasma & 34 versus 15 & $-84.54(-101.64,-67.44)$ & & \\
\hline SMD $(95 \%$ CI $)$ & TAS & Plasma & 73 versus 41 & $-43.30(-123.21,36.60)$ & $98.9 \%$ & $<0.0001$ \\
\hline Cervellati [34] & TAP & Serum & 56 versus 38 & $-0.22(-0.63,0.19)$ & & \\
\hline Yousefzadeh [23] & TAP & Plasma & 22 versus 22 & $-0.62(-1.22,-0.01)$ & & \\
\hline Cervellati [6] & TAP & Serum & 30 versus 63 & $0.04(-0.40,0.48)$ & & \\
\hline Sharma [32] & TAP & Serum & 35 versus 30 & $-13.18(-15.53,-10.83)$ & & \\
\hline SMD $(95 \% C I)$ & TAP & Serum/plasma & 142 versus 150 & $-2.74(-4.60,-0.87)$ & $97.5 \%$ & $<0.0001$ \\
\hline Ouzzif [27] & $\mathrm{VB}_{12}$ & Plasma & 58 versus 64 & $-0.13(-0.48,0.23)$ & & \\
\hline Bozkurt [28], & $\mathrm{VB}_{12}$ & Serum & 38 versus 48 & $0.05(-0.37,0.48)$ & & \\
\hline Haliloglu [29] & $\mathrm{VB}_{12}$ & Serum & 25 versus 53 & $-0.19(-0.66,0.29)$ & & \\
\hline Baines [30] & $\mathrm{VB}_{12}$ & Serum & 110 versus 110 & $-0.08(-0.34,0.19)$ & & \\
\hline Cagnacci [31] & $\mathrm{VB}_{12}$ & Serum & 28 versus 72 & $0.46(0.02,0.90)$ & & \\
\hline SMD $(95 \%$ CI) & $V B_{12}$ & Plasma/serum & 259 versus 347 & $-0.00(-0.20,0.21)$ & $29.6 \%$ & 0.224 \\
\hline Haliloglu [29] & Folate & Serum & 25 versus 53 & $-0.46(-0.94,0.02)$ & & \\
\hline Baines [30] & Folate & Serum & 110 versus 110 & $-0.39(-0.66,-0.12)$ & & \\
\hline Cagnacci [31] & Folate & Serum & 28 versus 72 & $-5.52(-6.41,-4.64)$ & & \\
\hline Bozkurt [28] & Folic Acid & Serum & 25 versus 53 & $0.04(-0.39,0.46)$ & & \\
\hline Ouzzif [27] & Folate & Plasma & 58 versus 64 & $-0.24(-0.60,0.11)$ & & \\
\hline Akpolat [24] & Folate & Serum & 66 versus 60 & $-1.03(-1.40,-0.66)$ & & \\
\hline$S M D(95 \% C I)$ & Folate & Serum/plasma & 325 versus 407 & $-1.18(-2.04,-0.31)$ & $96.4 \%$ & $<0.0001$ \\
\hline Akpolat [24] & Hcy & Plasma & 66 versus 60 & $0.09(-0.26,0.44)$ & & \\
\hline Yilmaz [22] & Hcy & Plasma & 34 versus 15 & $0.25(-0.36,0.86)$ & & \\
\hline Ouzzif [27] & Hcy & Plasma & 58 versus 64 & $0.51(0.14,0.87)$ & & \\
\hline Bozkurt [28] & Hcy & Serum & 38 versus 48 & $0.43(-0.00,0.88)$ & & \\
\hline Haliloglu [29] & Hcy & Serum & 25 versus 53 & $1.15(0.64,1.65)$ & & \\
\hline Baines [30] & Hcy & Plasma & 110 versus 110 & $0.24(-0.03,0.50)$ & & \\
\hline Cagnacci [31] & Hcy & Serum & 28 versus 72 & $1.22(0.75,1.68)$ & & \\
\hline$S M D(95 \% C I)$ & Hcy & Plasma/serum & 359 versus 422 & $0.53(0.23,0.84)$ & $75.3 \%$ & $<0.0001$ \\
\hline
\end{tabular}


that Hcy played an important role in bone metabolism and had been involved in osteoporotic facture incidence [41-43]. Our results indicated that Hcy was associated with the risk of $\mathrm{PO}$, which was in accordance with the previous clinical reports $[13,17,27,44,45]$ and also a recent meta-analysis [46]. Although inverse [47], mixed [48], and no associations $[31,49]$ between BMD and Hcy have also been reported, as Gerdhem et al. [47] reported, this controversial association between Hcy and BMD could be explained partly by the inability of the BMD measurement to reflect the status of bone metabolism. After all, all the results suggest the import role of Hcy in bone metabolism and the development of PO.

$\mathrm{VB}_{12}$ is essential for folate cycling and is known to be a determinant of total Hcy concentration. Our results revealed no significant change of $\mathrm{VB}_{12}$ in $\mathrm{PO}$ group compared with healthy control, which was not in agreement with a previous meta-analysis on the relationship between $\mathrm{VB}_{12}$ and $\mathrm{PO}$ [46]. This may be the reason why we exclude a paper written in Chinese. In addition, previous studies indicated that the increased $\mathrm{VB}_{12}$ levels failed to show a beneficial role for osteoporosis in PO $[28,30]$, which further reflected the dynamic process of osteoporosis. The genetic differences in the studied populations or the small study/sample size for meta-analysis may have contributed to the final conclusion; thus, larger clinical and long-term studies would be needed.

Folate status is another determinant of total Hcy concentration. In line with the results of Hcy in PO, a significantly decreased folate level appeared in PO groups in our metaanalysis. Although a similar trend was shown in Zhang H's study, no statistical difference was obtained in their metaanalysis [46]. This could be due to the update of the metaanalysis and the enlarged number of subjects included.

In the present meta-analysis, we investigated possible causes of heterogeneity among studies. Host factors can be ruled out because most studies were matched by age and gender and the studies were only carried out on PO subjects. Meanwhile, we pooled only measurements carried out on the same (or similar) biological sample and with reasonably comparable methods; thus, in a few cases, the heterogeneity persisted. In addition, all the OS markers and antioxidant system were taken into consideration in our meta-analysis, which made a solid foundation for comprehensive evaluation of the relationship between OS and PO. However, there are several limitations of the present study. The positive results achieved with OS markers are, however, flawed by the small sample size and lack of evidence that these molecules actually exerted an antioxidant effect in vivo. Secondly, although we attempted to consider as many confounding factors as possible, we cannot exclude the possibility that the observed associations could be attributed to uncontrolled factors that affect the condition of OS, such as 25-hydroxyvitamin D levels or years since menopause. Thirdly, the study population was mostly Turks and Italians, hence we could not be certain that our results will be applicable in other populations. Clinical trials in the future should be carried out to further test whether these biomarkers could be the "gold standard" for diagnostics and prevention.

In summary, our meta-analysis suggests a significant association between OS and PO. The imbalance of ROS and antioxidant system may contribute to functional and structural remodeling that favors the occurrence of PO. Despite many efforts made to effectively diagnose and therapeutically prevent PO occurrence, the results with several antiosteoporotic agents are not well satisfactory. Scavenging ROS overproduction or regulation of antioxidants activity could be investigated to see whether this may represent a novel therapeutic approach to prevent PO occurrence.

\section{Competing Interests}

The authors declare that they have no competing interests.

\section{Authors' Contributions}

Qiaozhen Zhou and Li Zhu contributed equally to this work.

\section{Acknowledgments}

The authors thank Dr. Chunjie Li for his great help with metaanalysis techniques and language polishing. This work was supported by the Natural Science Foundation of China (nos. 81271186, 81500817), Zhejiang Provincial Natural Science Foundation of China (no. LY15H140008), Health Science and Technology Project of Zhejiang Province (2016KYB184), and Wenzhou Public Technical Research Medical Program (Y20140708).

\section{References}

[1] M. N. Weitzmann and R. Pacifici, "Estrogen deficiency and bone loss: an inflammatory tale," Journal of Clinical Investigation, vol. 116, no. 5, pp. 1186-1194, 2006.

[2] J. Iwamoto, Y. Sato, T. Takeda, and H. Matsumoto, "Whole body vibration exercise improves body balance and walking velocity in postmenopausal osteoporotic women treated with alendronate: Galileo and Alendronate Intervention Trail (GAIT)," Journal of Musculoskeletal Neuronal Interactions, vol. 12, no. 3, pp. 136-143, 2012.

[3] D. L. Diab and N. B. Watts, "Postmenopausal osteoporosis," Current Opinion in Endocrinology, Diabetes and Obesity, vol. 20, no. 6, pp. 501-509, 2013.

[4] S. Das and J. C. Crockett, "Osteoporosis-a current view of pharmacological prevention and treatment," Drug Design, Development and Therapy, vol. 7, pp. 435-448, 2013.

[5] J. A. Kanis and O. Johnell, "Requirements for DXA for the management of osteoporosis in Europe," Osteoporosis International, vol. 16, no. 3, pp. 229-238, 2005.

[6] C. Cervellati, G. Bonaccorsi, E. Cremonini et al., "Bone mass density selectively correlates with serum markers of oxidative damage in post-menopausal women," Clinical Chemistry and Laboratory Medicine, vol. 51, no. 2, pp. 333-338, 2013.

[7] S. C. Manolagas, "From estrogen-centric to aging and oxidative stress: a revised perspective of the pathogenesis of osteoporosis," Endocrine Reviews, vol. 31, no. 3, pp. 266-300, 2010.

[8] S. Yang, D. Feskanich, W. C. Willett, A. H. Eliassen, and T. Wu, "Association between global biomarkers of oxidative stress and hip fracture in postmenopausal women: a prospective study," Journal of Bone and Mineral Research, vol. 29, no. 12, pp. 25772583, 2014 
[9] J. Flatow, P. Buckley, and B. J. Miller, "Meta-analysis of oxidative stress in schizophrenia," Biological Psychiatry, vol. 74, no. 6, pp. 400-409, 2013.

[10] X.-C. Bai, D. Lu, J. Bai et al., "Oxidative stress inhibits osteoblastic differentiation of bone cells by ERK and NF- $\kappa$ B," Biochemical and Biophysical Research Communications, vol. 314, no. 1, pp. 197-207, 2004.

[11] N. Mody, F. Parhami, T. A. Sarafian, and L. L. Demer, "Oxidative stress modulates osteoblastic differentiation of vascular and bone cells," Free Radical Biology and Medicine, vol. 31, no. 4, pp. 509-519, 2001.

[12] M. Almeida, L. Han, M. Martin-Millan et al., "Skeletal involution by age-associated oxidative stress and its acceleration by loss of sex steroids," The Journal of Biological Chemistry, vol. 282, no. 37, pp. 27285-27297, 2007.

[13] M. A. Sánchez-Rodríguez, M. Ruiz-Ramos, E. Correa-Muñoz, and V. M. Mendoza-Núñez, "Oxidative stress as a risk factor for osteoporosis in elderly Mexicans as characterized by antioxidant enzymes," BMC Musculoskeletal Disorders, vol. 8, article 124, 2007.

[14] S. Basu, K. Michaëlsson, H. Olofsson, S. Johansson, and H. Melhus, "Association between oxidative stress and bone mineral density," Biochemical and Biophysical Research Communications, vol. 288, no. 1, pp. 275-279, 2001.

[15] D. Maggio, M. Barabani, M. Pierandrei et al., "Marked decrease in plasma antioxidants in aged osteoporotic women: results of a cross-sectional study," Journal of Clinical Endocrinology and Metabolism, vol. 88, no. 4, pp. 1523-1527, 2003.

[16] A. N. Sontakke and R. S. Tare, "A duality in the roles of reactive oxygen species with respect to bone metabolism," Clinica Chimica Acta, vol. 318, no. 1-2, pp. 145-148, 2002.

[17] S. Yalin, S. Bagis, S. C. Aksit, H. Arslan, and C. Erdogan, "Effect of free radicals and antioxidants on postmenopausal osteoporosis," Asian Journal of Chemistry, vol. 18, no. 2, pp. 1091-1096, 2006.

[18] S. Ozgocmen, H. Kaya, E. Fadillioglu, and Z. Yilmaz, "Effects of calcitonin, risedronate, and raloxifene on erythrocyte antioxidant enzyme activity, lipid peroxidation, and nitric oxide in postmenopausal osteoporosis," Archives of Medical Research, vol. 38, no. 2, pp. 196-205, 2007.

[19] I. Dalle-Donne, R. Rossi, R. Colombo, D. Giustarini, and A. Milzani, "Biomarkers of oxidative damage in human disease," Clinical Chemistry, vol. 52, no. 4, pp. 601-623, 2006.

[20] C. N. Black, M. Bot, P. G. Scheffer, P. Cuijpers, and B. W. J. H. Penninx, "Is depression associated with increased oxidative stress? A systematic review and meta-analysis," Psychoneuroendocrinology, vol. 51, pp. 164-175, 2015.

[21] O. Altindag, O. Erel, N. Soran, H. Celik, and S. Selek, “Total oxidative/anti-oxidative status and relation to bone mineral density in osteoporosis," Rheumatology International, vol. 28, no. 4, pp. 317-321, 2008.

[22] N. Yilmaz and E. Eren, "Homocysteine oxidative stress and relation to bone mineral density in post-menopausal osteoporosis," Aging Clinical and Experimental Research, vol. 21, no. 4-5, pp. 353-357, 2009.

[23] G. Yousefzadeh, B. Larijani, A. Mohammadirad et al., "Determination of oxidative stress status and concentration of TGF- $\beta 1$ in the blood and saliva of osteoporotic subjects," Annals of the New York Academy of Sciences, vol. 1091, pp. 142-150, 2006.

[24] V. Akpolat, H. M. Bilgin, M. Y. Celik, M. Erdemoglu, and B. Isik, "An evaluation of nitric oxide, folate, homocysteine levels and lipid peroxidation in postmenopausal osteoporosis," Advances in Clinical and Experimental Medicine, vol. 22, no. 3, pp. 403409, 2013.

[25] O. F. Sendur, Y. Turan, E. Tastaban, and M. Serter, "Antioxidant status in patients with osteoporosis: a controlled study," Joint Bone Spine, vol. 76, no. 5, pp. 514-518, 2009.

[26] M. Zinnuroglu, A. S. Dincel, F. Kosova, V. Sepici, and G. K. Karatas, "Prospective evaluation of free radicals and antioxidant activity following 6-month risedronate treatment in patients with postmenopausal osteoporosis," Rheumatology International, vol. 32, no. 4, pp. 875-880, 2012.

[27] Z. Ouzzif, K. Oumghar, K. Sbai, A. Mounach, E. M. Derouiche, and A. El Maghraoui, "Relation of plasma total homocysteine, folate and vitamin B12 levels to bone mineral density in Moroccan healthy postmenopausal women," Rheumatology International, vol. 32, no. 1, pp. 123-128, 2012.

[28] N. Bozkurt, M. Erdem, E. YIlmaz et al., "The relationship of homocyteine, B12 and folic acid with the bone mineral density of the femur and lumbar spine in Turkish postmenopausal women," Archives of Gynecology and Obstetrics, vol. 280, no. 3, pp. 381-387, 2009.

[29] B. Haliloglu, F. B. Aksungar, E. Ilter, H. Peker, F. T. Akin, and U. Ozekici, "Relationship between bone mineral density, bone turnover markers and homocysteine, folate and vitamin B12 levels in postmenopausal women," Archives of Gynecology and Obstetrics, vol. 281, no. 4, pp. 663-668, 2010.

[30] M. Baines, M.-B. Kredan, J. Usher et al., "The association of homocysteine and its determinants MTHFR genotype, folate, vitamin B12 and vitamin B6 with bone mineral density in postmenopausal British women," Bone, vol. 40, no. 3, pp. 730736, 2007.

[31] A. Cagnacci, F. Baldassari, G. Rivolta, S. Arangino, and A. Volpe, "Relation of homocysteine, folate, and vitamin B12 to bone mineral density of postmenopausal women," Bone, vol. 33, no. 6, pp. 956-959, 2003.

[32] T. Sharma, N. Islam, J. Ahmad, N. Akhtar, and M. Beg, "Correlation between bone mineral density and oxidative stress in postmenopausal women," Indian Journal of Endocrinology and Metabolism, vol. 19, no. 4, pp. 491-497, 2015.

[33] Q. Wu, Z.-M. Zhong, Y. Pan et al., "Advanced oxidation protein products as a novel marker of oxidative stress in postmenopausal osteoporosis," Medical Science Monitor, vol. 21, pp. 2428-2432, 2015.

[34] C. Cervellati, G. Bonaccorsi, E. Cremonini et al., "Oxidative stress and bone resorption interplay as a possible trigger for postmenopausal osteoporosis," BioMed Research International, vol. 2014, Article ID 569563, 8 pages, 2014.

[35] X. Wang, W. Wang, L. Li, G. Perry, H.-G. Lee, and X. Zhu, "Oxidative stress and mitochondrial dysfunction in Alzheimer's disease," Biochimica et Biophysica Acta (BBA)-Molecular Basis of Disease, vol. 1842, no. 8, pp. 1240-1247, 2014.

[36] H. Sies, "Strategies of antioxidant defense," European Journal of Biochemistry, vol. 215, no. 2, pp. 213-219, 1993.

[37] N. I. Krinsky, "Mechanism of action of biological antioxidants," Proceedings of the Society for Experimental Biology and Medicine, vol. 200, no. 2, pp. 248-254, 1992.

[38] C. Michiels, M. Raes, O. Toussaint, and J. Remacle, "Importance of se-glutathione peroxidase, catalase, and $\mathrm{Cu} / \mathrm{Zn}$-SOD for cell survival against oxidative stress," Free Radical Biology and Medicine, vol. 17, no. 3, pp. 235-248, 1994.

[39] R. J. van't Hof and S. H. Ralston, "Nitric oxide and bone," Immunology, vol. 103, no. 3, pp. 255-261, 2001. 
[40] J. Aguirre, L. Buttery, M. O’Shaughnessy et al., "Endothelial nitric oxide synthase gene-deficient mice demonstrate marked retardation in postnatal bone formation, reduced bone volume, and defects in osteoblast maturation and activity," The American Journal of Pathology, vol. 158, no. 1, pp. 247-257, 2001.

[41] M. S. Leboff, R. Narweker, A. Lacroix et al., "Homocysteine levels and risk of hip fracture in postmenopausal women," The Journal of Clinical Endocrinology \& Metabolism, vol. 94, no. 4, pp. 1207-1213, 2009.

[42] R. R. McLean, P. F. Jacques, J. Selhub et al., "Homocysteine as a predictive factor for hip fracture in older persons," The New England Journal of Medicine, vol. 350, no. 20, pp. 2042-2049, 2004.

[43] J. B. J. van Meurs, R. A. M. Dhonukshe-Rutten, S. M. F. Pluijm et al., "Homocysteine levels and the risk of osteoporotic fracture," The New England Journal of Medicine, vol. 350, no. 20, pp. 20332041, 2004.

[44] M. S. Morris, P. F. Jacques, and J. Selhub, "Relation between homocysteine and B-vitamin status indicators and bone mineral density in older Americans," Bone, vol. 37, no. 2, pp. 234242, 2005.

[45] J. Golbahar, A. Hamidi, M. A. Aminzadeh, and G. R. Omrani, "Association of plasma folate, plasma total homocysteine, but not methylenetetrahydrofolate reductase C667T polymorphism, with bone mineral density in postmenopausal Iranian women: a cross-sectional study," Bone, vol. 35, no. 3, pp. 760$765,2004$.

[46] H. Zhang, X. Tao, and J. Wu, "Association of homocysteine, vitamin B12, and folate with bone mineral density in postmenopausal women: a meta-analysis," Archives of Gynecology and Obstetrics, vol. 289, no. 5, pp. 1003-1009, 2014.

[47] P. Gerdhem, K. K. Ivaska, A. Isaksson et al., "Associations between homocysteine, bone turnover, BMD, mortality, and fracture risk in elderly women," Journal of Bone and Mineral Research, vol. 22, no. 1, pp. 127-134, 2007.

[48] P. Bucciarelli, G. Martini, I. Martinelli et al., "The relationship between plasma homocysteine levels and bone mineral density in post-menopausal women," European Journal of Internal Medicine, vol. 21, no. 4, pp. 301-305, 2010.

[49] M. A. Périer, E. Gineyts, F. Munoz, E. Sornay-Rendu, and P. D. Delmas, "Homocysteine and fracture risk in postmenopausal women: the OFELY study," Osteoporosis International, vol. 18, no. 10, pp. 1329-1336, 2007. 


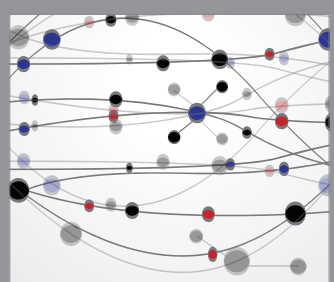

The Scientific World Journal
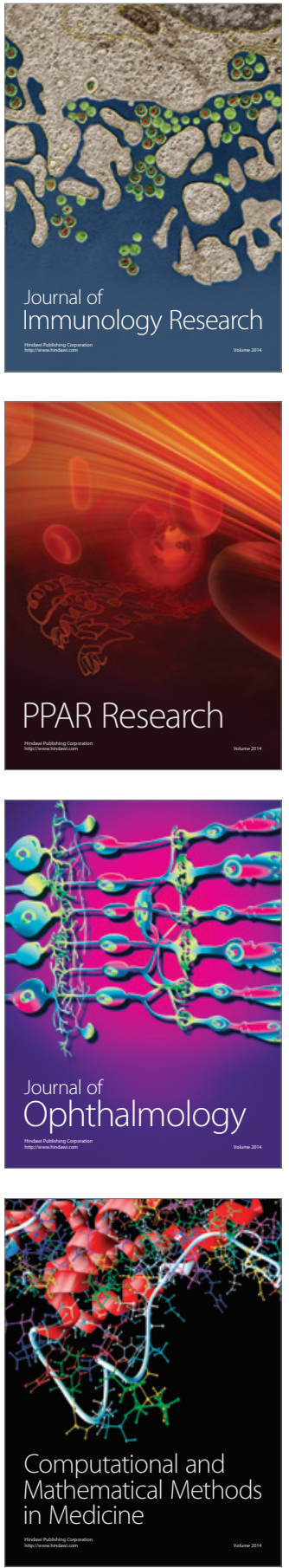

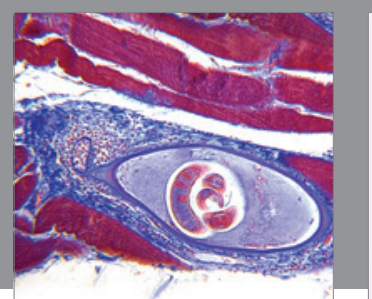

Gastroenterology Research and Practice

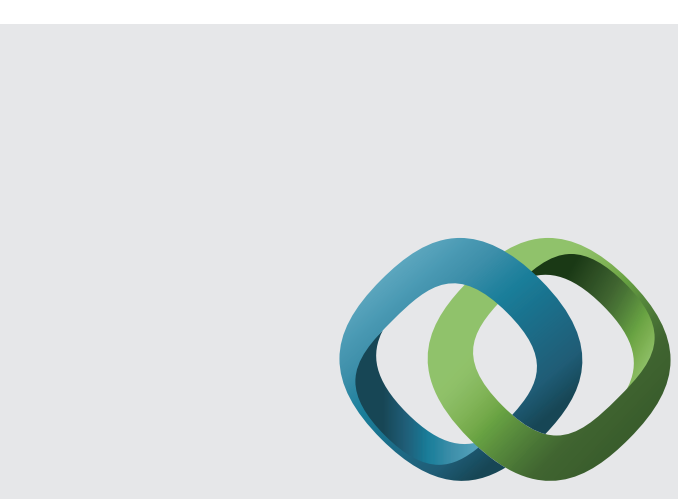

\section{Hindawi}

Submit your manuscripts at

http://www.hindawi.com
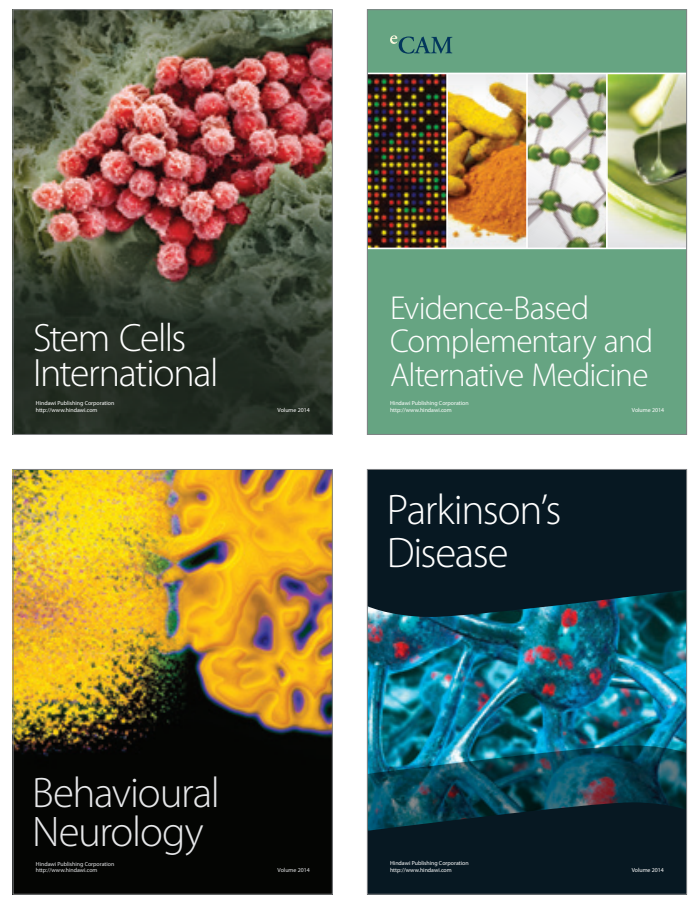
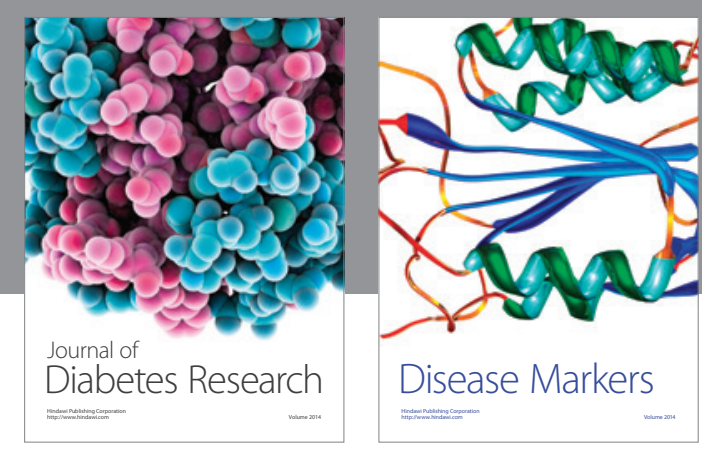

Disease Markers
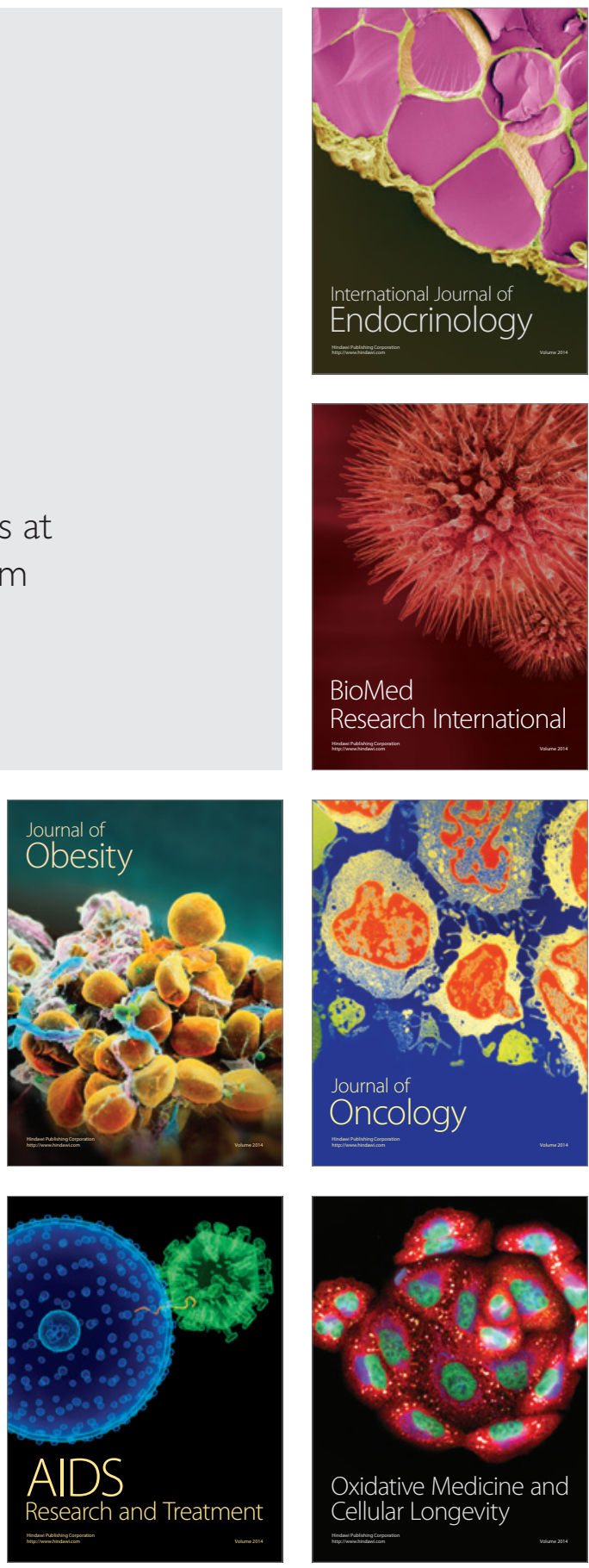